ISSN: 2527-1075

\title{
VINHO DE LARANJA (Citrus sinensis L. Osbeck): UM ESTUDO COM DIFERENTES LINHAGENS DE Saccharomyces cerevisiae
}

\author{
ORANGE WINE (Citrus sinensis L. Osbeck): A STUDY WITH DIFFERENT LINES \\ OF Saccharomyces cerevisiae
}

\author{
V. C. ALCÂNTARA ${ }^{1}$; E. G. T. MENEZES ${ }^{1}$ \\ Universidade Federal de Viçosa - Campus Rio Paranaíba \\ E-mail: ctrvalter@gmail.com
}

a rticle info

Article history:

Received 12 May 2017

Accepted 3 August 2017

Available online 20 September 2017
PALAVRAS-CHAVE: Fermentação alcoólica; Laranja; Leveduras.

KEYWORDS: Alcoholic fermentation; Orange; Yeasts.

\begin{abstract}
RESUMO: Diversos fatores influenciam na obtenção de bebidas fermentadas, como a escolha da matériaprima, ponto de maturação dos frutos e o processo fermentativo. Sendo assim, o objetivo deste trabalho foi comparar e avaliar o processo fermentativo de três linhagens de Saccharomyces cerevisiae, utilizando suco de laranja como substrato. As variedades estudadas foram: CA 11, PE 2 e fermento biológico FERMIX ${ }^{\circledR}$. Na pesquisa, foi realizado o acompanhamento das fermentações, observando o consumo dos açúcares, $p H$, teores alcoólicos finais, acidez, rendimento e os açúcares residuais. Os fermentados apresentaram teores alcoólicos entre 9,5 a $12,3{ }^{\circ} \mathrm{GL}$, sendo o menor para a o fermento FERMIX ${ }^{\circledR}$ e o maior para cepa industrial PE 2. A Linhagem CA 11 apresentou menor tempo para o consumo dos açúcares dos mostos. A fermentação mais lenta foi apresentada pela $\underline{\text { S. cerevisiae } P E}$ 2, entretanto essa levedura apresentou maior eficiência e rendimento, 94 $\%$ e 0,485 $\mathrm{g} \mathrm{g}^{-1}$, respectivamente. A acidez total teve seu menor índice para a cepa PE 2 (102,1 meq $\left.\mathrm{L}^{-1}\right)$, e todas encontraram-se dentro dos padrões estabelecidos por legislação para bebidas fermentadas. Os fermentados foram classificados como vinho de laranja meio seco, visto que os teores de açúcares residuais totais foram superiores a 5, $1 \mathrm{~g} \mathrm{L-1,} \mathrm{onde} \mathrm{o} \mathrm{menor} \mathrm{teor} \mathrm{foi} \mathrm{observado} \mathrm{na} \mathrm{linhagem} \mathrm{CA} 11 \mathrm{com} \mathrm{6,6} \mathrm{g} \mathrm{L^{-1 }}$. Desta forma conclui-

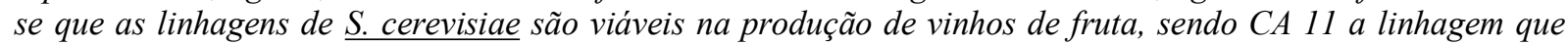
apresentou processo mais rápido, bom rendimento e elevado teor alcoólico final.
\end{abstract}

\footnotetext{
ABSTRACT: Several factors influence the obtaining of fermented beverages, such as the choice of raw material, fruit maturation point and fermentation process. Thus, the objective of this work was to compare and evaluate the fermentation process of three strains of Saccharomyces cerevisiae, using orange juice as substrate. The varieties studied were: $C A 11, P E 2$ and FERMIX® biological yeast. In the research, fermentation was monitored, observing the sugars consumption, pH, final alcohol content, acidity, yield and residual sugars. The fermentation had alcoholic contents between 9.5 and $12.3^{\circ} \mathrm{GL}$, being the lowest for the fermentation FERMIX® and the larger for industrial strain PE 2. The Lineage CA 11 presented less time for the consumption of the sugars of the musts. The slower fermentation was presented by $S$. cerevisiae PE 2, however this yeast presented higher efficiency and yield, 94\% and $0.485 \mathrm{~g} \mathrm{~g}^{-1}$, respectively. The total acidity had its lowest index for the PE 2 strain $\left(102.1 \mathrm{meq} \mathrm{L^{-1 }}\right)$, and all were within the standards established by legislation for fermented beverages. Fermentation was classified as dry half orange wine, as the total residual sugars contents were higher than $5.1 \mathrm{~g}$ $L^{-1}$, where the lowest content was observed in the CA 11 line with $6.6 \mathrm{~g} \mathrm{~L}^{-1}$. In this way, it is concluded that $\underline{\underline{S}}$. cerevisiae strains are viable in the production of fruit wines, with CA 11 being the line that presented a faster process, good yield and high final alcohol content.
} 


\section{The Journal of Engineering and Exact Sciences - \\ JCEC}

ISSN: 2527-1075

\section{INTRODUÇÃO}

A produção de fermentados alcoólicos é um dos mais antigos processos, possivelmente iniciado com a produção de vinho e cerveja há milhares de anos (VENTURINI, 2010). As bebidas fermentadas de frutas constituem produtos economicamente viáveis, além de contribuírem para a redução de perdas pós-colheita de frutos perecíveis (MUNIZ et al., 2002).

O Brasil dispõe de uma grande diversidade de frutas com produção praticamente por todo o ano, como laranja (Citrus sinensis), e apresenta a maior capacidade produtiva deste fruto no mundo, seguido pelos Estados Unidos, China, Índia e México (SANTOS et al., 2005).

Segundo a legislação brasileira, fermentado de fruta é definido como uma bebida com graduação alcoólica que varia entre $4 \%$ a $14 \%$ em volume $\left(20{ }^{\circ} \mathrm{C}\right)$ e deve ser obtido pela fermentação alcoólica do mosto da fruta sã, fresca e madura de uma única espécie, do respectivo suco integral ou concentrado, ou polpa (BRASIL, 2009).

Na produção de vinhos o uso de leveduras como a Saccharomyces cerevisiae viabiliza uma série de vantagens, tais como: fermentação completa e regular, maior produção de etanol, possibilidade de uma clarificação mais rápida e melhoria da sua estabilidade biológica (TONET, 2007). A utilização de linhagens selecionadas proporciona maior tolerância às condições de estresse encontradas durante a fermentação, como: elevadas concentrações de sacarose, altas temperaturas, concentrações elevadas de etanol e variações de pH (BARBOSA, 2013).

Neste contexto, o presente estudo buscou comparar o processo fermentativo de mosto de laranja de três linhagens de S. cerevisiae, a fim de se avaliar qual levedura mais adequada à produção de vinhos de frutas, obtendo um processo com maior eficiência e rendimento.

\section{MATERIAL E MÉTODOS}

Os fermentados de laranja foram produzidos no laboratório de processamento de alimentos da Universidade Federal de Viçosa campus Rio Paranaíba. A matéria prima, laranja (Citrus sinensis L. Osbeck) foi obtida em mercado local da cidade de Rio Paranaíba - Minas Gerais. Foram utilizadas três estirpes de leveduras, sendo elas: $S$. cerevisiae CA 11, S. cerevisiae PE 2 e fermento FERMIX ${ }^{\circledR}$. O processamento das bebidas seguiu as etapas apresentadas pela Figura 1.

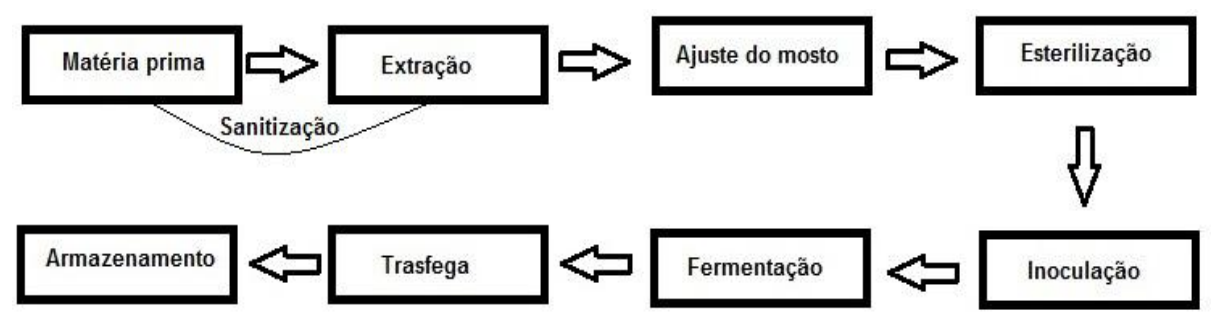

Figura 1 - Etapas de processamento dos fermentados de laranja.

A quantidade de fermento utilizada foi definida com base nos valores obtidos de contagem de células viáveis em câmara de Neubauer (LEE, et al. 1981), no intuito de 


\section{The Journal of Engineering and Exact Sciences - \\ JCEC \\ ISSN: 2527-1075}

adicionar cerca de 1,6 x 109 células viáveis por tratamento, conferindo homogeneização entre os tratamentos. Os valores obtidos nas análises do suco de laranja e as quantidades de leveduras utilizadas estão apresentados na Tabela 1.

Tabela 1 - Dados do suco das laranjas e a quantidade de fermento adicionado

\begin{tabular}{lccc}
\hline Tratamentos & ${ }^{\circ}$ Brix (suco) & pH (suco) & Levedura (g) \\
\hline Laranja (CA 11) & 8,5 & $4,03^{ \pm 0,19}$ & 22,0 \\
Laranja (PE 2) & 8,7 & $3,98^{ \pm 0,31}$ & 22,5 \\
Laranja (FERMIX ${ }^{\circledR}$ ) & 8,3 & $4,01^{ \pm 0,20}$ & 12,5 \\
\hline
\end{tabular}

As fermentações foram realizadas em triplicata utilizando erlenmeyers de 1 litro, contendo $500 \mathrm{~mL}$ de mosto, em B.O.D Eletrolab, modelo EL 202, com temperatura controlada a $25{ }^{\circ} \mathrm{C}$. Durante o período de fermentação, foram retiradas amostras nos tempos específicos $(0,6,22,28,45$ e 51 horas), para realização das análises. Ao final do processo de fermentação realizou-se a trasfega do vinho, o qual foi engarrafado e armazenado sobre refrigeração.

Foi realizado o acompanhamento das fermentações, analisando $\mathrm{pH}$ e sólidos solúveis ( ${ }^{\circ}$ Brix). Os fermentados obtidos foram submetidos a análises de acidez, pelo método de titulometria utilizando $\mathrm{NaOH} 0,1 \mathrm{~N}$ e indicador de fenolftaleína, análise da densidade por picnometria a $20^{\circ} \mathrm{C}$ e teor alcoólico, utilizando tabela de conversão da densidade relativa para graduação alcoólica a $20{ }^{\circ} \mathrm{C}$. Estas análises foram realizadas em triplicata e seguiram o manual de bebidas fermentadas do Instituto Adolfo Lutz (2008). Os açúcares totais foram determinados pelo método de Fehling, seguindo os princípios utilizados por Tavares et al. (2010), onde os açúcares totais é corresponde a soma dos açúcares redutores e não redutores, expressos em glicose e sacarose.

\section{RESULTADOS E DISCUSSÃO}

As fermentações ocorreram dentro do tempo estimado e sem aparecimento de contaminação. Todas as bebidas apresentaram teores alcoólicos dentro dos padrões estabelecidos pela legislação para bebidas fermentadas, sendo estes de $4 \%$ a $14 \%(\mathrm{v} / \mathrm{v})$ (Tabela 2) (BRASIL, 2008).

Tabela 2 - Resultados das análises no produto final

\begin{tabular}{lccc}
\hline Fermento & CA 11 & PE 2 & FERMIX $^{\circledR}$ \\
\hline Teor alcoólico $\left({ }^{\circ} \mathrm{GL}\right)$ & $10,4 \pm, 1,5$ & $12,3 \pm 0,1$ & $9,5 \pm 0,1$ \\
ART $\left(\mathrm{g} 100 \mathrm{~mL}^{-1}\right)$ & $0,66 \pm 0,02$ & $0,78 \pm 0,08$ & $0,89 \pm 0,05$ \\
Rendimento $\left(\mathrm{g} \mathrm{g}^{-1}\right)$ & $0,410 \pm 0,006$ & $0,485 \pm 0,008$ & $0,374 \pm 0,005$ \\
Eficiência $(\%)$ & $80 \pm 0,5$ & $94 \pm 0,8$ & $73 \pm 0,6$ \\
Acidez total (meq L-1) & $125,5 \pm 0,1$ & $102,1 \pm 2,6$ & $114,2 \pm 4,8$ \\
Estabilização (h) & 22 & 45 & 28 \\
\hline
\end{tabular}

A fermentação utilizando a cepa PE 2 apresentou maior teor alcoólico $\left(12,3{ }^{\circ} \mathrm{GL}\right)$ e uma eficiência de $94 \%$. Resultado esperado, visto que esta variedade de $S$. cerevisiae é destinada à 


\section{The Journal of Engineering and Exact Sciences - \\ JCEC \\ ISSN: 2527-1075}

produção de álcool combustível e apresenta maior resistência as variações de $\mathrm{pH}$ e ao teor de álcool do meio, sendo esta de até $15 \%$ (v/v) (LNF, 2009). Basso et al. (2008) analisando a fermentação de caldo e melaço de cana-de-açúcar (Saccharum officinarum), observaram elevada eficiência de processo (92 \%) utilizando a cepa PE 2. Em estudo de hidrolisado de batata-doce (Ipomoea batatas) Povlak et al. (2011) observaram que a S. cerevisiae PE 2 apresentou eficiência superior a linhagem de fermento de panificação, sendo os valores iguais a $97,1 \%$ e $91,1 \%$, respectivamente.

O fermento FERMIX ${ }^{\circledR}$ apresentou menor produção em álcool $\left(9,5^{\circ} \mathrm{GL}\right)$. Uma vez que esta variedade é destinada à panificação, sua eficiência (73\%) para conversão do substrato em produto é menor quando comparada às demais leveduras utilizadas. Lopes (2006) trabalhando com fruto da palma forrageira (Opuntia ficus-indica Mill), obteve uma eficiência de fermentação de $72 \%$, utilizando levedura de panificação.

Ribeiro (2014) analisando bebida fermentada de caldo de cana e abacaxi (Ananas comosus (L.) Merril), encontrou uma eficiência de 83,9 \% e um rendimento de 0,433 gramas de etanol para cada grama de substrato, utilizando $S$. cerevisiae CA 11, valor próximo ao obtido neste estudo com a variedade CA 11, onde obtivemos $80 \%$ e 0,410 , para eficiência e rendimento, respectivamente.

As estabilizações das fermentações ocorreram para CA $11 \mathrm{em} 22 \mathrm{~h}$, FERMIX ${ }^{\circledR}$ em $28 \mathrm{~h}$ e $45 \mathrm{~h}$ para a variedade PE 2, determinada pela consolidação do teor de sólidos solúveis (Figura 2). Os teores de açúcares residuais apresentaram valores semelhantes, sendo o menor encontrado para CA $11\left(0,66 \mathrm{~g} 100 \mathrm{~mL}^{-1}\right)$. Souza (2011) encontrou teor de $0,9 \mathrm{~g} 100 \mathrm{~mL}^{-1} \mathrm{de}$ açúcares residuais totais em fermentado de maçã (Pyrus malus L) de cultivar Gala, utilizando a cepa CA 11.

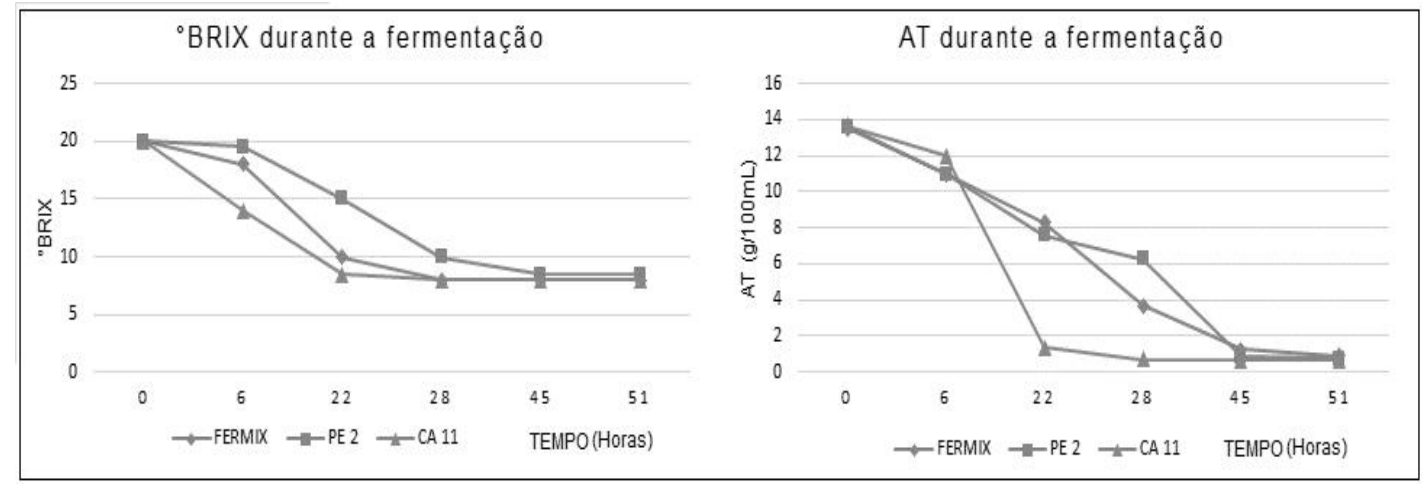

Figura 2 - Relação da queda de ${ }^{\circ}$ Brix com o consumo de açúcares totais durante o processo fermentativo.

Os fermentados deste estudo são classificados como vinhos de fruto meio secos, visto que todos apresentaram concentrações de açúcares totais residuais superiores a $5,1 \mathrm{~g} \mathrm{~L}^{-1} \mathrm{e}$ inferiores a $20 \mathrm{~g} \mathrm{~L}^{-1}$, segundo a legislação brasileira (BRASIL, 1988), a qual estabelece as classes de bebidas referentes à quantidade de açúcares residuais. $\mathrm{O}$ fermentado obtido pela variedade CA 11 apresentou maior acidez total com 125,5 meq L $\mathrm{L}^{-1}$ e a PE 2 apresentou menor índice de acidez com 102,1 meq $\mathrm{L}^{-1}$. Todas as bebidas estão dentro dos padrões da legislação, a qual estabelece limites de 50 a $130 \mathrm{meq} \mathrm{L}^{-1}$ (BRASIL 2008). 


\section{The Journal of Engineering and Exact Sciences - \\ JCEC}

ISSN: 2527-1075

A variação do pH durante a fermentação ficou entre 4,01 e 4,12, sendo provinda da liberação de ácidos orgânicos pelas leveduras no decorrer do processo fermentativo, como o succínico, acarretando o decréscimo no $\mathrm{pH}$. Por outro lado, o consumo de ácidos presentes no mosto após o esgotamento dos açúcares ocasionou seu acréscimo.

\section{CONCLUSÕES}

Conclui-se que apesar das diferentes especificações de fabricação das leveduras utilizadas, todas apresentaram-se viáveis na produção de fermentados de frutas, produzindo bebidas com boas características físico-químicas. A variedade CA 11 demonstrou menor tempo para estabilização da fermentação, bom rendimento e consumo quase total dos açúcares presentes no mosto. O fermento FERMIX ${ }^{\circledR}$ demostrou resultados relevantes, como a velocidade de conversão do substrato em produto superior à da cepa $\mathrm{PE} \mathrm{2,} \mathrm{entretanto}$ apresentou eficiência muito inferior, em relação a esta cepa, a qual é destinada à indústria de álcool.

\section{REFERÊNCIAS}

BARBOSA, E. A. Caracterização molecular e bioquímica de linhagens de Saccharomyces cerevisiae da região de Salinas para fins de identificação geográfica. 2013. 143p. Tese de Doutorado (Doutor em Ciências Biológicas), Universidade Federal de Ouro Preto, Ouro Preto, 2013.

BASSO, L. C. AMORIM, H. V. Oliveira, A. J.; LOPES, M. L. Yest selection for fuel etanol production in Brazil. FEMS Yeast Research, vol. 8, 2008.

BRASIL. Decreto $\mathbf{n}^{\mathbf{0}}$. 6.871, de 04 de Junho de 2009. Regulamenta a Lei no 8.918, de 14 de julho de 1994, que dispõe sobre a padronização, a classificação, o registro, a inspeção, a produção e a fiscalização de bebidas, 2009.

BRASIL. Portaria n. 64 de 23 de abril de 2008. Aprovam os regulamentos técnicos para a fixação dos padrões de identidade e qualidade para as bebidas alcoólicas fermentadas. Brasília, Ministério da Agricultura, Pecuária e Abastecimento, 2008.

BRASIL. Portaria n. ${ }^{\circ}$ 229, de 25 de outubro de 1988. Complementação de padrões de identidade e qualidade de vinho. Ministério da Agricultura Pecuária e Abastecimento. Brasília, 1988.

LEE, S.S.; ROBINSON, F.M.; WANG, H.Y. Rapid determination of yeast viability. Biotechnology Bioengineering Symposium, v.11, p.641-649, 1981.

LOPES, R. V. V. L.; SILVA, F. L. H. Elaboração de fermentados a partir do figo-da-india. Revista de biologia e ciências da terra, vol. 6, n. 2, 2006.

LUTZ, A. INSTITUTO ADOLFO LUTZ. Métodos físicos químicos para análises de alimentos. Procedimentos e determinações gerais. Editora Instituto Adolfo Lutz, cap. 4, p. 83-160, 2008.

MUNIZ, C. R. et al. Bebidas fermentadas a partir de frutos tropicais. Boletim do CEPPA, v. 20, n. 2, 2002.

PAVLAK, M. C. M.; ABREU LIMA, T. L.; CARREIRO, S. C. Estudo da fermentação do hidrolisado de batata-doce utilizando diferentes linhagens de Saccharomyces cerevisiae. Química Nova, vol. 34, n. 1, 2011. 
RIBEIRO, L. S. Elaboração de bebida fermentada de caldo de cana e abacaxi utilizando leveduras Saccharomyces e não Saccharomyces. 88f. 2014. Dissertação (Mestre em Microbiologia Agrícola). Universidade Federal de Lavras, Lavas, 2014.

SANTOS, S. C. et al. Elaboração e análise sensorial do fermentado de acerola (Malpighia punicifolia L.). Braz. J. Food Technol., Campinas, v. 10, p. 47-50, 2005.

TAVARES, J. T. Q.; CARDOSO, R. L.; COSTA, J. A.; FADIGAS, F. S.; FONSECA, A. A. Interferência do ácido ascórbico na determinação de açúcares redutores pelo método de Lane e Eynon. Química Nova, vol. 33, n. 4, 2010.

TONET, A. Avaliação de Quatro Leveduras para a Produção de Espumante pelo Método Champenoise. Monografia (Tecnologia em Viticultura e Enologia). Centro Federal de Educação Tecnológica de Bento Gonçalves, Bento Gonçalves, 2007.

VENTURINI, W. G. F. Bebidas alcoólicas: ciência e tecnologia. 1.ed. São Paulo: Editora Blucher, 85p. 2010. 